\title{
DIFFRACTION GRATING STRUCTURES IN SOLAR CELLS
}

\author{
Saleem H. Zaidi ${ }^{1}$, James M. Gee ${ }^{2}$, and Douglas S. Ruby ${ }^{2}$ \\ 'Gratings, Inc., Albuquerque, NM 87107 \\ ${ }^{2}$ Sandia National Laboratories, Albuquerque, NM 87185-0752
}

\begin{abstract}
Sub-wavelength periodic texturing (gratings) of crystalline-silicon (c-Si) surfaces for solar cell applications can be designed for maximizing optical absorption in thin c-Si films. We have investigated c-Si grating structures using rigorous modeling, hemispherical reflectance, and internal quantum efficiency measurements. Model calculations predict almost $\sim 100 \%$ energy coupling into obliquely propagating diffraction orders. By fabrication and optical characterization of a wide range of 1D \& 2D c-Si grating structures, we have achieved broadband, low $(\sim 5 \%)$ reflectance without an anti-reflection film. By integrating grating structures into conventional solar cell designs, we have demonstrated short-circuit current density enhancements of 3.4 and $4.1 \mathrm{~mA} / \mathrm{cm}^{2}$ for rectangular and triangular $1 \mathrm{D}$ grating structures compared to planar controls. The effective path length enhancements due to these gratings were 2.2 and 1.7, respectively. Optimized $2 \mathrm{D}$ gratings are expected to have even better performance.
\end{abstract}

\section{INTRODUCTION}

In most $\mathrm{c}$-Si photovoltaic modules, $50 \%$ of the cost is associated with the c-Si wafer [1]. Therefore, a highly effective approach aimed at cost reduction in crystalline and multi-crystalline c-Si solar cells is better material utilization by using thinner $(\sim 1-20 \mu \mathrm{m})$ films. Reduced film thickness is also attractive due to lower bulk recombination and increased open-circuit voltages provided complete optical confinement can be achieved [2]. c-Si is strongly absorptive in the UV-visible spectral region. However, in near IR region $(\sim 0.8-1.1 \mu \mathrm{m})$ and particularly near the band edge, the absorption is weak; e.g. the absorption depth is $\sim 100 \mu \mathrm{m}$ at $\lambda \sim 1 \mu \mathrm{m}$ [3]. This weak absorption fundamentally limits the performance of thin-film c-Si solar cells. Surface texturing schemes aimed at enhancing near IR absorption by oblique optical coupling into the semiconductor have been extensively investigated [4]. Surface structuring schemes based on geometrical optics considerations may have limited applicability in thin films due to texture dimension approaching the film thickness.

A periodically textured surface such as a grating structure can be used to enhance absorption in thin films. Sheng, et al. first proposed this for thin film a-Si solar cells [5]. Using a waveguide configuration, it was shown that an appropriately designed grating structure can enhance short-circuit current by $\sim 4-6 \mathrm{~mA} / \mathrm{cm}^{2}$ in comparison with a planar surface. Heine and Morf investigated diffraction gratings in c-Si solar cells [6]. In their scheme, grating structures were designed for optimum coupling into obliquely propagating diffraction orders inside the semiconductor material. Broadband antireflection characteristics of grating structures have also been extensively investigated [7].

Here, we report on an investigation of submicrometer c-Si grating structures by modeling, hemispherical reflectance, and internal quantum efficiency measurements. This investigation will help in optimizing grating structures aimed at maximizing optical absorption in thin-film c-Si solar cell devices.

\section{MODELING OF GRATING STRUCTURES}

Light interaction with a grating structure is a complex function of several parameters including incident wavelength, angle, polarization, period, profile, duty cycle, and depth [8]. For solar cell applications, the frontsurface grating should be designed to enhance infrared absorption by efficiently coupling of light into the modes that propagate nearly parallel to the surface while minimizing coupling into the normally propagating zeroorder mode. Using commercially available GSOLVER ${ }^{\mathrm{TM}}$ modeling software, we have investigated rectangular, triangular, and blazed grating structures.

Figure 1 is an example calculation. It shows the diffraction efficiency for both TE and TM-polarizations for normal incidence. The grating period was selected at $0.65 \mu \mathrm{m}$ so that both first and second orders were propagating. Finer periods - e.g., $d=0.32 \mu \mathrm{m}$, where only the two \pm 1 -orders relatively weak energy coupling. For the TE-polarization, $\sim 40 \%$ of the incident energy at $0.5 \mu \mathrm{m}$ depth is coupled into the \pm 1 -diffraction orders (propagating at $\sim 30^{\circ}$ with respect to the normal), and minimal energy is coupled into the \pm 2 -diffraction orders (propagating at $\sim 60^{\circ}$ with respect to the normal). For TM-polarization, almost $80 \%$ of the incident energy is coupled to the \pm 1 and \pm 2 -diffraction orders. Similar calculations for triangular-profiled grating structure show that at TE-polarization, $\sim 60 \%$ of incident energy is coupled into the \pm 1 -diffraction orders and approximately $10 \%$ is coupled into the \pm 2 -diffraction orders. At TMpolarization, $-80 \%$ of the energy is coupled into \pm 1 diffraction orders and $\sim 10 \%$ into the \pm 2 -diffraction orders. Finally, for the blazed grating profiles, nearly $100 \%$ of the incident energy is coupled into diffraction orders for the TE polarization. For TM-polarization, same behavior is seen with $\sim 30 \%$ coupling into the +2 diffraction order. A comparison of the energy distribution 


\section{DISCLAIMER}

This report was prepared as an account of work sponsored by an agency of the United States Government. Neither the United States Government nor any agency thereof, nor any of their employees, make any warranty, express or implied, or assumes any legal liability or responsibility for the accuracy, completeness, or usefulness of any information, apparatus, product, or process disciosed, or represents that its use would not infringe privately owned rights. Reference herein to any specific commercial product, process, or service by trade name, trademark, manufacturer, or otherwise does not necessarily constitute or imply its endorsement, recommendation, or favoring by the United States Government or any agency thereof. The views and opinions of authors expressed herein do not necessarily state or reflect those of the United States Government or any agency thereof. 


\section{DISCLAIMER}

Portions of this document may be illegible in electronic image products. Images are produced from the best available original document. 
among diffraction orders for the three profiles reveals that the blazed grating structure couples more energy into the transmitted diffraction orders, but that coupling into the second order is comparable for the three grating structures. The rectangular profile shows the least energy transfer into diffraction orders.

In general, our calculations show that high diffraction efficiencies can be achieved as long as the propagation angle is $\leq 60^{\circ}$ with respect to the surface normal. For higher propagation angles, most of the energy is coupled back into the normally propagating zero-order modes.
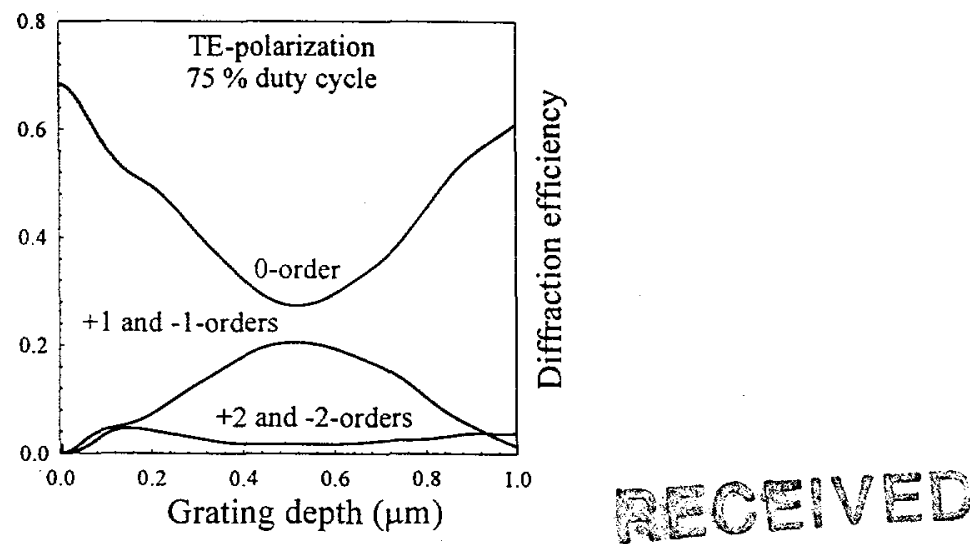

DEC 222090

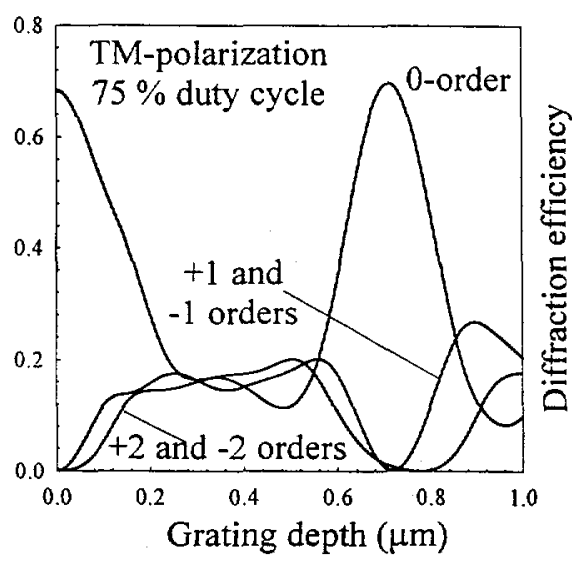

QS SI
Figure 1. Diffraction efficiency calculations as a function of grating depth for symmetric rectangular profile grating structure at $\lambda=1.0 \mu \mathrm{m}$.

\section{FABRICATION OF GRATING STRUCTURES}

We have developed low-cost, large-area interferometric lithography techniques to pattern a wide range of 1D and 2D periodic structures [9]. Interference between two coherent laser beams produces a simple periodic pattern at $d=\lambda / 2 \sin \theta$, where $\lambda$ is the exposure wavelength, and $2 \theta$ is the angle between the two intersecting laser beams. For $\lambda=355 \mathrm{~nm}, \theta=60^{\circ}$, periods down to $\sim 200$-nm can be easily fabricated. Typically, grating structures are first formed in photoresist followed by pattern transfer to c-Si using an appropriate combination of wet and dry etching techniques. Figure 2 shows scanning electron microscope pictures of ID triangular and rectangular profile gratings, and a 2D hole pattern etched in c-Si using RIE and wet-chemical etching methods [10]. The triangular structure shown in Fig. 2 is characterized by period of $\sim 420 \mathrm{~nm}$ with full width at half-maximum depth of $\sim 100 \mathrm{~nm}$ etched to a depth of $600 \mathrm{~nm}$. The rectangular profile grating shown in Fig. 1 has a period of $500 \mathrm{~nm}$, linewidth of $\sim 130 \mathrm{~nm}$, and depth of $\sim 1000 \mathrm{~nm}$. The 2D hole pattern at $800-\mathrm{nm}$ period is has a linewidth $\sim 270 \mathrm{~nm}$ and depth of $\sim 800 \mathrm{~nm}$.

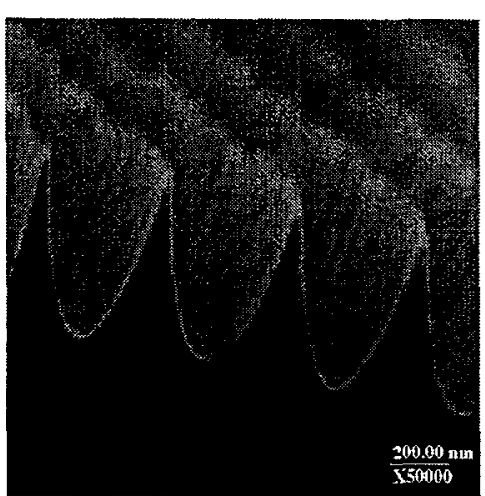

(a)



(b)

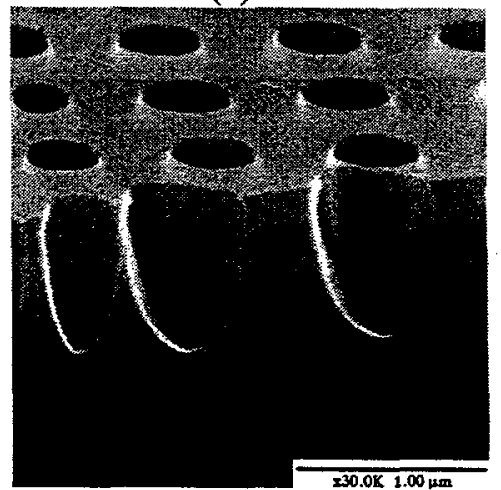

Figure 2. SEM photomicrographs of (a) 1D triangular, (b) 1D rectangular, and (c) 2D hole pattern grating.

Spectral reflectance measurements of grating structures were carried out using a Cary Model 5E (UVVIS-NIR) spectrophotometer and a Labsphere (RSA-CA- 
50) integrating sphere. Figure 3 shows absolute hemispherical reflectance measurements from grating structures shown in Fig. 2. It is seen that broadband reduced reflection is observed from the triangular grating profile. In contrast, the rectangular-profiled grating structure supports a number of narrow, low reflectance spectral bands. For the triangular profiles, the same broadband anti-reflection behavior is seen with both polarizations. For the rectangular profiles, these spectral bands are a critical function of the incident light polarization. For the $2 \mathrm{D}$ hole-pattern, a broadband anti-reflection behavior is observed. The reflection can be further reduced in all cases by application of anti-reflection films.

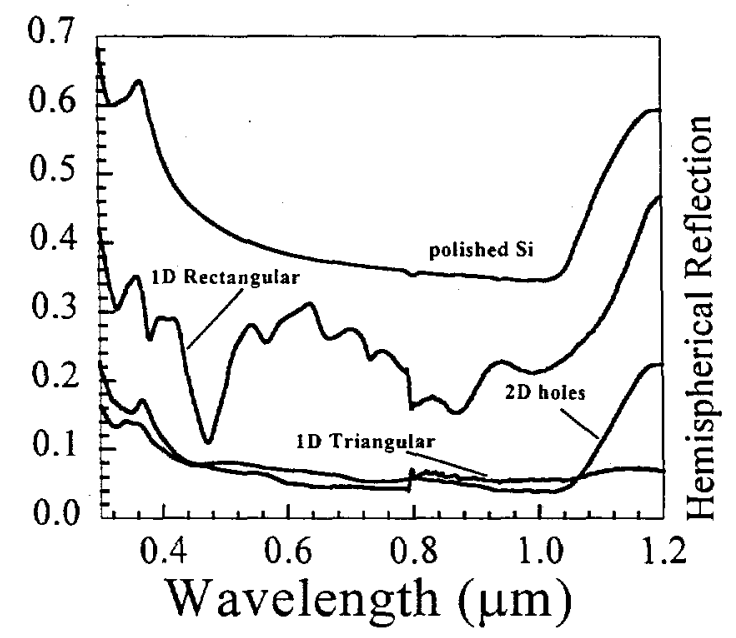

Figure 3. Hemispherical reflectance measurements from grating structures shown in Fig. 2. Reflectance from polished c-Si surface is shown for comparison.

\section{INTERNAL QUANTUM EFFICIENCY MEASUREMENTS}

Grating solar cells were fabricated on $4^{\prime \prime}$ diameter, p-type, $1 \Omega$-cm resistivity wafers at Sandia National Laboratories. Wafers were RIE textured with periodic patterns. The junction depth was $\sim 0.5 \mu \mathrm{m}$ on the planar region. A 10 -nm thick passivating $\mathrm{SiO}_{2}$ film was grown during back surface field formation at $-900{ }^{\circ} \mathrm{C}$. For comparison, planar and grating regions of $\sim 2 \times 2 \mathrm{~cm}^{2}$ were formed on each cell. Internal quantum efficiency measurements from planar and grating regions were acquired to understand the influence of grating structure on cell efficiency. The IQE measurements of triangular and rectangular profiled 1D gratings exhibited substantially reduced blue response. This was attributed to the lack of adequate passivation in the strongly absorptive grating region, and probably to some residual surface damage suffered from the RIE process. By incorporating $\mathrm{RCA}$ clean process prior to emitter formation, the blue response was significantly improved. Figure 4 shows the IQE response of a $1 D$ rectangular profile grating structure. For comparison, the planar surface IQE adjacent to the grating is also plotted. It is seen that the grating structure cell still exhibits lower IQE in $0.4-0.6-\mu \mathrm{m}$ spectral region presumably due to residual RIE-induced surface damage. The IQE response, however, is significantly enhanced in the long wavelength region. The surface reflectance has also been significantly reduced.

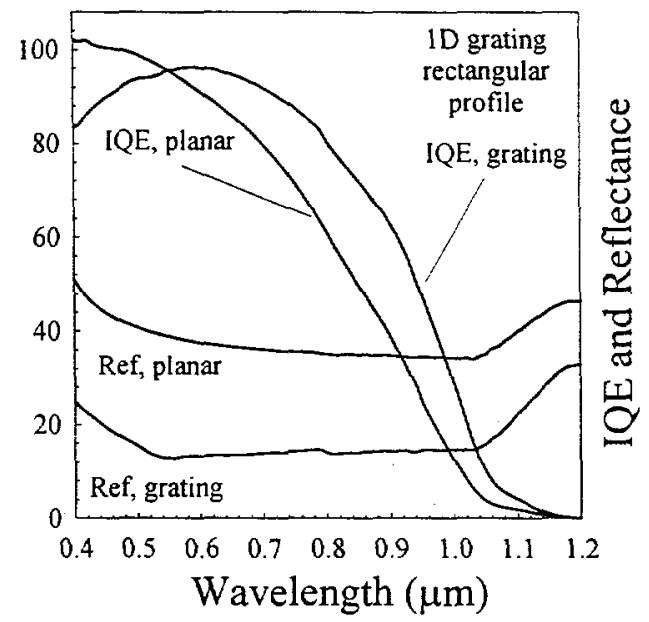

Figure 4. Internal quantum efficiency from 1D rectangular profile grating. For comparison, IQE response of planar surface is also shown.

Figure 5 shows similar IQE and reflectance measurements from a ID triangular profiled grating structure. This grating structure exhibits slightly improved IQE response in the short wavelength region, but it is still lower than the planar surface. The enhancement in the long wavelength region is not as pronounced as for the rectangular profile.

We can eliminate the substrate variation by plotting IQE ratios from the grating and planar regions of the same wafer. Fig. 6 plots the IQE ratios for the rectangular and triangular profiled grating structures. It is seen that for the rectangular profile, the ratio is less than 1 for wavelengths $<0.55 \mu \mathrm{m}$, and the maximum enhancement of $\sim 2.7$ is observed at $\lambda \sim 1.1 \mu \mathrm{m}$. For the triangular profile, the IQE ratio is less than 1 for wavelengths $<0.5 \mu \mathrm{m}$, while the maximum enhancement of $\sim 1.6$ is seen at $\lambda \sim 1.1 \mu \mathrm{m}$. The IQE enhancement in the IR region can best be understood on the basis of the diffraction calculations presented earlier. The grating structure couples significant energy into the obliquely propagating diffraction orders inside $\mathrm{c}$-Si. These transmitted diffraction orders generate electron-hole pairs closer to the surface, thus, enhancing the probability of collection by the junction.

\section{ANALYSIS OF RESULTS}

There is clearly an improvement in IQE due to the grating (Fig. 4-6). A convenient method to quantify 
the effect of the grating alone on improving IQE is to analyze the inverse IQE versus absorption length [11]. The linear regime of this relationship in the near infrared region gives a measure of the internal collection length ( $\mathrm{L}_{\text {iqe }}$ and Fig. 7). $\mathrm{L}_{\mathrm{iqe}}$ is a function of the bulk material quality (e.g., bulk diffusion length) and the internal path length. Since the planar and grating regions were adjacent within the same cell and have very similar bulk properties, a ratio of $L_{\text {iqe }}$ between the textured and planar regions gives the effective increase in path length due to the grating. We refer to "effective" path length enhancement since the generation profile with the grating is a combination of different modes traveling at different angles. The effective path length enhancement for the triangular and rectangular profiles is roughly 1.7 and 2.2 , respectively. For comparison, the path length enhancement with chemically textured surfaces is around 1.3 since the light is coupled into the silicon at a well-defined angle [11].



Figure 5. Internal quantum efficiency from ID triangular profile grating. For comparison, IQE response of planar surface is also shown.

\section{ACKNOWLEDGEMENTS}

Gratings, Inc. was partially supported for this work by Sandia National Laboratories under contract BE8229. The authors would like to thank Samuel McCormack, Bev Silva, Jeannette Moore, and Barry Hansen for help with cell fabrication and measurements. Sandia National Laboratories is a multiprogram laboratory operated by Sandia Corporation, a Lockheed Martin Company, for the U. S. Department of Energy under contract DE-ACO4-94AL85000.

\section{REFERENCES}

[1] A. Suzuki, M. Kaneiwa, T. Saga, and S. Matsuda, IEEE Trans. Elect. Dev., ED-46, 2126 (1999).

[2] T. Tiedje, E. Yablonovitch, G. D. Cody, and B. G. Brooks, IEEE Trans. Elect. Dev., ED-31, 711 (1984).
[3] M. A. Green and M. J. Keevers, Prog. in Photo. Research and Applications 3, 189 (1995).

[4] P. Campbell and M. A. Green, Appl. Phys. Lett. 62, 243 (1987).

[5] Ping Sheng, A. N. Bloch, and R. S. Stepleman, Appl. Phys. Lett. 43, 579 (1983).

[6] C. Heine and R. H. Morf, Appl. Opt. 34, 2476 (1995).

[7] D. H. Raguin and G. M. Morris, Appl. Opt. 32, 1154 (1993).

[8] S. Kemme, S.H. Zaidi, and J. M. Gee, $9^{\text {th }}$ Workshop on c-Si Mat. and Proc., Breckenridge (1999).

[9] Saleem H. Zaidi and S. R. J. Brueck, J. Vac. Sci. Technol. B11, 458 (1993).

[10] Saleem H. Zaidi, An-Shyang Chu, and S. R. J. Brueck, J. Appl. Phys. 80, 6997 (1996).

[11] Paul A. Basore, 23 ${ }^{\text {rd }}$ IEEE Photo. Spec. Conf., 147 (1993).



Figure 6. Ratio of IQE response from grating to the planar surfaces for rectangular and triangular profiles.

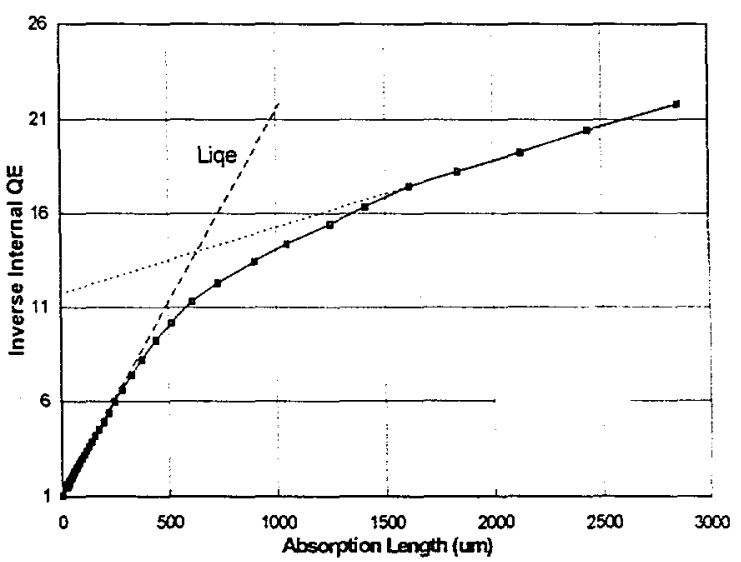

Figure 7. Plot of inverse-IQE versus photon absorption length for the planar cell of Fig. 5 . $\mathrm{L}_{\text {iqe }}$ is the inverse slope of the initial linear regime [11]. 